\title{
Optimization Techniques Incorporating Evolutionary Model in Wireless Sensor Network: A Survey
}

\author{
Vidya Honguntikar ${ }^{1}$, Dr. G. S. Biradar ${ }^{2}$ \\ ${ }^{I}$ (Department of Telecommunication, Dr. Ambedkar Institute of Technology, India) \\ ${ }^{2}$ (Department of Electronics and Communication, P.D.A. College of Engineering, India)
}

\begin{abstract}
Wireless Sensor Network has several issues and challenges that are to be taken care while designing the techniques and algorithms to increase the Network lifetime of WSN. Network optimization is a critical component and optimization techniques are used to achieve the design goals in Networking. With several optimization algorithms existing to suit different problems, choosing a proper algorithm is very important in any optimization technique. In this paper we present the summary of different optimization techniques incorporating Evolutionary model and their approach in the different areas of Wireless sensor Network. These evolutionary techniques are studied from the Environment and are used to incorporate similar behavior in Wireless Sensor Networks. This Paper includes the discussion of few other papers which uses such Evolutionary model in different areas of optimization.
\end{abstract}

Keywords: Energy Efficiency, Evolutionary model, Network Lifetime, Optimization technique, Wireless sensor Network.

\section{Introduction}

Wireless sensor network (WSN) is a structure of spatially distributed sensor nodes for gathering significant information about the target environment. It is a large collection of small wireless devices that are spatially dispersed all over the region to be monitored and they systematize themselves to form an Ad hoc network which is capable of performing collaborative work for holding the general functionality. The battery powering with integrated sensors, data processing capabilities with short range radio communication convey the sensor nodes which are so called as small wireless devices.

WSN is built with few to thousands of nodes, where each node is connected to a sensor. Each such sensor network node has a radio transceiver with internal antenna or external antenna, a microcontroller, an electronic circuit for interfacing with sensors and an energy source which is a battery. WSN applications can be categorized as Tracking and Monitoring applications. Tracking includes Enemy tracking, Animal tracking, Human tracking, or Traffic tracking. Monitoring involves security detection in Military, Animal monitoring like zebra, birds, cane toad etc., Inventory monitoring in Business, Industrial monitoring, Patient monitoring in health and finally Environmental monitoring of weather, temperature and pressure [1]. WSNs have incredible research interest due to their recent applications in Civil and Military field. Intellectual gathering of sensor data, transmission and controlling depending on the applications is intrinsic in such systems. Latent ability to observe and manipulate the physical environment via network of small sensors outfitted with processor, memory and communication assets is provided by WSN.

The objective of this paper is to present an overview of WSN Optimization using the Evolutionary models and to discuss the existing papers related in this field. Section II deals with the major challenges of WSN. In section III we highlight the need for optimization in WSN. Section IV is presented with a brief description of Optimization techniques incorporating the Evolutionary models. Section V gives a brief description of related papers and finally in Section VI we conclude this paper.

\section{Challenges in WSN}

The wireless sensor network has its essential features and technical challenges. Some of the features of WSNs are limited memory, node failure, mobility, low computing capability and constrained resources like bandwidth, power and communication resources. WSN are engaged in a variety of real time fields like military, disaster, agriculture, medical etc. especially in the areas of need for efficient techniques, protocols and intellectual algorithm. Some potential issues and challenges exist when we are designing the techniques and algorithms. Some of them are discussed below.

- Routing: In sensor network the sensed data is to be routed from multiple sensor nodes to sink, via the most suitable path taking into account the resource constraints of WSN. Thus routing is a challenging concern here.

- Time Synchronization: In order to fuse data effectively, various levels of data integration are desirable at various levels and should be synchronized, as data is propagated towards the sink. 
- Localization: For obtaining the accuracy in data reception and location, the complexity increases in large scale networks for complex environments. The challenge here is to find the location of each sensor node, its data with less communication and processing cost.

- Security and Privacy: From catastrophic attacks, wireless connected nodes embedded in the environment cannot be ensured. Node network can be modified, replaced with malicious counterpart, and in making observation the sensors can be fooled by attackers which do not precisely reflect the environment.

- Scalability: Are deployed with hundreds, thousands or more sensor nodes, based on the applications requirement and the region to be monitored. With such large number of wireless connected sensor nodes, there is requirement of techniques and their computations to be scalable enough to respond and operate in real time.

- Energy Conservation: In WSN, development of new secure and energy efficient methods enabling fusion of relatively large amount of data is the basic requirements of data processing. Over a massively Distributed database with constraint resources, data aggregation/data fusion has to be performed. The accuracy reflection of the state of the environment in final, high level sensing results delivered by the System, the sensors deployment event timing, insufficiency of energy and bandwidth to record all the raw data surety also will be considerable.

\section{Need For Optimization in WSN}

Optimization is the act, process or methodology of obtaining the best result under a given circumstances. The word optimum is taken to mean 'maximum' or 'minimum' depending on circumstances. Network optimization is a critical component and the optimization techniques are used to achieve design goals in networking. Energy efficiency, cost and application requirement are the challenges that are to be taken care while designing a WSN. This requires optimization of both hardware and software to make WSN efficient. Software addresses issue of Network Lifetime [1].

There are several optimization algorithms to suit the different problems. Choosing a proper algorithm is very important in any optimization technique. With a very little infrastructure for WSN, the deployment of the sensor nodes is either inside the monitoring area or very near to it [8]. Batteries used in sensor nodes are impossible to replace or recharge since the sensor nodes are deployed in remote/hostile sensing areas. Thus, end of a battery life in a node essentially means the end of network itself. Efficient use of battery energy is hence crucial to enhance the network lifetime. The sensor network protocols have to focus primarily on power conservation issues. Other issues are, achieving high quality QoS, low bandwidth, limited processing and storage in every node [2]. These are the issues which are directly related to the problem of optimization.

\section{Optimization Techniques Incorporating Evolutionary Model}

The evolutionary techniques are studied from the environment and are used to incorporate similar behavior in WSN to improve their Network lifetime, QoS and other desired WSN properties that need the right choice of optimizer or algorithm. The possible evolutionary techniques that are adopted are based on Bio-Mimic optimization strategies. Some of the optimization algorithms incorporating evolutionary model and swarm Intelligence are Particle Swarm optimization (PSO), Ant colony optimization (ACO), BEES optimization Algorithm (BOA), Particle Swarm Frog leaping hybrid optimization algorithm, Elephant swarm optimization, Cuckoo search (CS), Bat Algorithm (BA,), Firefly Algorithm (FA) and Genetic Algorithm (GA).

\section{Particle Swarm optimization (PSO)}

This is a computational method to optimize a problem by iteratively trying to improve a candidate solution for a given measure of quality. PSO algorithm works by having a population called Swarm of candidate's solution called particles. The movements of particles are guided by their own best known position in search-space as well as the entire swarm's position which in turn guide the movement of swarm and the same is repeated until a satisfactory solution is achieved. PSO is a multidimensional optimization technique which is used in Node deployment, Localization, Energy aware clustering and Data aggregation [2].

\section{Ant Colony Optimization}

This is a very good probabilistic technique to solve computational problems which can be reduced to finding good paths through graphs. Foraging behavior of some Ant species is the inspiration for ACO method. These Ants leave a trail of pheromone which is a secreted or excreted chemical hormone that triggers a social response in members of the same species on the ground in order to mark their paths from the nest to food that should be followed by other members of the colony [2]. The food foraging behavior of Ants is as follows:

- The first ant finds the food source, and then returns to the nest, leaving behind a pheromone trail. 
- All other ants indiscriminately follow possible ways, and the strengthening of the runway will arrive at shortest route.

- Ants take the shortest route and lose their trail pheromones in case of long routes.

This algorithm helps in solving discrete optimization problems in various domains of Engineering. ACO is more flexible and decentralized in nature and also there is a similarity between Ants foraging and Routing. Thus the dynamic and distributed problems of routing in networks can be solved using ACO[2].

\section{BEES Optimization Algorithm}

This is a population-based optimization algorithm that imitates the food foraging behavior of swarm of honey Bees. In this algorithm, a kind of neighborhood search combined with random search is performed and can be used for both functional optimization and combinational optimization [3]. Generally Colony of honey bees faces an optimization problem during its search for food since it has to explore long distances as well as different directions to get a large amount of food sources. To execute this task, the colony sends scout bees to search for food locations randomly. After finding the location of large food resources they return and pass on this information to the colony through a dance called Waggle dance. This dance enables the colony to employ more bees to the large food resources and fewer bees for the lesser food resources. This approach leads to an efficient searching and harvesting. The colony continues to send scout bees to perform random search during food gathering season, and therefore it keeps its chance to find new food resources. Clustering of data, neural networks training and multi-objective optimization are the different applications in which this algorithm is applied.

\section{Frog Leaping optimization}

For Collision Free transmission scheduling in wireless communication, the calling behavior of frogs can be applied where in the frogs call their neighbors alternately. The frog calls by making a sound for a certain period of time and then quiets down before repeating the call. If two or more frogs call at random, the timing of their call overlap which leads to interference and this results in failure. Therefore, each male frog shifts the timing of its calls by listening to the calls of other frogs so as to avoid such overlap. After the establishment of interaction pattern, call alternation without interference is achieved within a group. Thus transmission scheduling with a phase control is inspired by Frog Calling Behavior [5].

Shuffled Frog leaping Algorithm is swarm intelligence based sub-heuristic computation optimization Algorithm proposed to solve discrete combinatorial optimization problem [6]. In the calling behavior of Japanese tree frogs, a frog stops calling once it detects the calls of other neighboring frogs. This behavior can be applied in the design of an energy-efficient sleep control mechanism which provides adaptive operation periods which helps to prolong the Network Life time [7].

\section{Elephant Swarm Optimization}

Elephants are social animals and exhibit advanced intelligence. Elephants are known for their good memory this enables them to survive and lead the clan. Their memory empowers them with recognition, identification and problem solving scenarios. Their nature to coexist, group leading and survive within a clan socially formulated during testing times like migration and when the resources are scare. The secret of their longevity is their exhibition of the unselfish behavior which enables them to grow. The older elephant disassociates themselves from their clan keeping progress and survivability in mind. Elephants group communicates using varied advanced techniques which include acoustic communication, chemical communication, visual communication, and tactile communication. All these features influence to incorporate such behavior in wireless sensor network to enhance their network lifetime [8]. The behavior of elephant which is very complex can be incorporated in WSN using Cross Layer approach in terms of improved network lifetime, reduced node energy decay rate, higher active node ratios and lower communication overheads.

\section{Genetic Algorithm(GA)}

This algorithm is based on abstract of Darwin's evolution of biological systems. Genetic Algorithm is categorized as a global search methodology. In order to reach and obtain a possible global optimum solution, Genetic Algorithm uses the random search in the decision space through selection, crossover and mutation operations. Another operator of Genetic Algorithm is elitism. Its job is to store the best or elite chromosome for the next generation. Genetic Algorithms are implemented and presented using simulations and these are transformed into an optimization problem [2]. The following are the two advantages of GA over traditional algorithms:

1. Capability of handling complex problems and parallelism. 
2. GA can deal with all sorts of objective functions whether they are stationary or transient, Linear or Nonlinear, Continuous or Discontinuous. Multiple genes can be suitable for parallel implementation.GA can be incorporated in WSN clustering, deployment, routing and aggregation.

\section{Related Papers}

In Paper [9], the authors explain the importance of energy consumption in research of sensor networks. The authors of this paper have explained the approach to sensor networks routing, based on energy consumption. They have used very special technique called Swarm intelligence. In short it is collective behavior of decentralized, self-organized systems, natural or artificial. This concept of Swarm intelligence is invented by Gerardo Beni and Jing Wang in 1989. The main objective of this paper was transferring messages with low energy using swarm algorithm.

The research paper [11] provides an artificial ant-colony move toward distributed sensor wakeup control in WSN to accomplish the combined task of surveillance and target tracking. Artificial Ants are multiagent methods inspired by the behavior of real ants. A WSN consists of spatially distributed autonomous sensors to monitor physical or environmental conditions. WSN has great potential in military and civil applications. This is first the biologically-inspired sensor wakeup control method in WSN. It has advantages that are listed below.

i) This is distributive sensor and it does not require a centralized control or cluster leaders. That is reason it is free of the errors caused by leader failures and can keep the message cost for leader choice.

ii) This is robust to wrong alarms because the pheromone is accumulated temporally and spatially and thus is more trustworthy or dependable for wakeup control.

Biological systems present notable adaptation, consistency and sturdiness in numerous environments, even under antagonism. They are controlled by the individuals in a distributed and self-prearranged way. These biological mechanisms are responsible for convenient resources for dynamic and adaptive routing arrangements of wireless mobile sensor networks, in which the distinct nodes should ideally function without significant control. The paper [12] inspects vital biologically inspired contrivances and the related techniques for determining routing in wireless sensor networks, together with Ant-based and genetic tactics. The authors present a mathematical model of the biological reckonings in the framework of sensor networks. They further extend indiscriminate routing framework in sensor networks by spreading diverse modes of biological reckonings using Ant-based and Genetic methods. Finally, an impression of several evolving research instructions are given within the new biologically computational framework.

In [5], the authors have majorly focused on Japanese tree frog which belongs to genus Hyla group. The adaptability and stimulation of Japanese tree frog are discussed in this research. With the help of this explanation, the authors have connected the above special feature with wireless networks. A self-organizing scheduling scheme inspired by the special frog calling behavior as a method for satisfying such requirement is also concentrated.

The paper [15] summarizes issues and introduces Particle swarm optimization and its suitable applications. Wireless sensor networks are the fastest growing technology which is having bundle of prospective application in surveillance, atmosphere, healthcare, calamity management. Wireless networks are self-prevailing nodes used for scrutinizing an environment. Researchers of Wireless Sensor Networks face problems in communication link breakdown, memory and computational controls, and energy. Multidimensional optimization problems are big issue in wireless sensor networks and move towards through bio-motivated techniques. The focal problems are deployment, localization, energy-aware clustering and data-aggregation. The optimization method requires reasonable memory and enviable results of computational resources. Particle swarm optimization is an effortless, effective and unproblematic implemented optimization algorithm.

Many algorithms have been discovered on topic of wireless sensor Networks. But the disadvantage is power consumption, because of the battery limitation in sensors. A sensor senses the change of the capacity and also sensing data, transfer the outcome to the intention node all the way through wireless communication. Batteries permitted by wireless sensors are somewhat limited. If the battery of a sensor is out of control, the communication between nodes will lose connection and the transferring data of the other nodes will be amplified. The authors of the paper[18] describe an Ant colony optimization (ACO) based routing algorithm to condense power consumption. An evaluation table is built and referred to produce possible routing paths. Then, the ACO looks at these paths to reduce the power consumption. The simulations indicate that the proposed algorithm obtains more balanced transmission among the nodes and reduces the power consumption of the routing.

In WSN applications, localization of the nodes is the most important key enabling role. Here, the authors of the paper [4] are using Bees Optimization Algorithm (BOA) for localizing the nodes of the wireless sensor networks. This algorithm performs a neighborhood search combined with random search based on the population which is motivated by the natural foraging behavior of honey bees. This paper deals with the minimization of the objective function which is based on the summation of the squared range error between the 
node and the anchors. Based on normal random distribution for time of arrival (TOA) measurements and lognormal distribution for received signal strength (RSS) measurements different simulation tests with different topologies have been performed. This approach leads to an efficient searching and harvesting. This algorithm is going to perform more searches in the neighborhood of the promising solutions, and also partially keeping the random search to avoid falling in a local maximum/minimum solution. This has applications in clustering of data, neural networks training and multi-objective optimization. Here the authors simulate the reference nodes to display the effect of the references geometry on the solution. The simulation is showing the estimated error closed to that of the CRB in case of TOA measurements in different reference nodes distribution. This RSS measurement follows log-normal distribution that on turn is going to compensate the relative accuracies from several reference nodes.

WSNs are challenging to implement on Efficient-Energy Coverage (EEC) due to the need of limited energy use. The authors of paper [20] recommend new methodology to solve the EEC problem using Ant Colony Optimization (ACO) algorithm. ACO algorithm has an exclusive characteristic that conventional ACO algorithms do not have. Conventional ACO algorithms use only one type of pheromone whereas proposed ACO algorithm (Three Pheromones ACO, TPACO) uses three types of pheromones to find result efficiently. One of them is the local pheromone, which assistances an ant unify its coverage set with fewer sensors, other two are global pheromones, one is used to optimize the number of required active sensors per Point of Interest, and the other is used to form a sensor set, that has as many sensors as an ant selected the number of active sensors by using the former pheromone. In TPACO algorithm two user constraints of ACO algorithms are not used. Some techniques that lead to a more representative methodology to solve the EEC problem are used. One is to utilize the probabilistic sensor detection model and the other to use different kinds of sensors, i.e. heterogeneous sensors in continuous space.

The WSNs having small nodes are embedded with sensing ability, calculation and connection. But these are having source restrictions on connection, calculation and energy consumption. Due to the limited energy in sensor networks, the optimized energy consumption in above networks is significant and results in many problems. However, the energy consumption can be reduced by organizing the network nodes in several clusters and hence the lifetime will be enhanced. We may use the Queen-bee algorithm to create energy efficient clusters in wireless sensor networks. The Queen-bee (QB) is analogous to nature in that the queen-bee is the important member in reproduction process. The QB is simulated using J-sim simulator, which has provided a complete protocol stack for the applications of the sensor networks and results in the clustering by the QB algorithm which is going to decrease the energy consumption and enhancing the lifetime of the network. In comparison of the conventional genetic algorithm, the Queen-bee evolution Algorithm has the major difference as here only a part of the individuals are mutated with normal mutation probability $\mathrm{p}_{\mathrm{m}}$ and the others are mutated with strong mutation probability $\mathrm{P}_{\mathrm{m}}{ }_{\mathrm{m}}$. Here, the mother Queen-bee is required and selected for the reproduction of bees and the Queen-bee is going to reproduce many children in addition to a number of the bee-population using the crossover operator, so the numbers of marriages in the Queen-bee algorithm are much lower. In order to solve the convergence problem the authors of paper [21] use the normal or strong mutation for diversity that results in avoiding the pre-mature divergence

\section{Conclusion}

To achieve the design goals of Networking, Network optimization becomes a critical component. A proper algorithm can be chosen that suits to solve the problem using Evolutionary methods studied from the Environment. Introducing to WSN, its application and challenges, we, in this paper have made an effort to present an overview of WSN optimization techniques and their area of optimization using Evolutionary methods studied from the environment to enhance the Network Lifetime of WSN. Further the Network Optimization can also be achieved by a Cross-layered approach using an Evolutionary model. In Cross-layer approach data is shared among layers which minimizes the transfer overhead and hence it is more effective and energy efficient when compared to traditional layered approach.

\section{References}

[1] Jennifer Yick, Biswanth Mukherjee, DipakGhosal,” Wireless Sensor network survey”, Computer networks (2008), 52, pp. 22922330.

[2] Md. Akhtaruzzaman Adnan, MohammdAbdurRazzaque, Ishtiaque Ahmed, Ismail Fauzi Is nin,” Bio-mimic Optimization Strategies in Wireless Sensor Networks: A Survey", Sensors 2014, 14, pp. 299-235.

[3] E.M. Saad, M.H. Awadalla, and R.R. Darwish, "Adaptive Energy-Aware Gathering Strategy for Wireless Sensor Networks", International journal of computers, Issue 2, volume 2, 2008, pp. 148-157.

[4] A. Moussa and N El-Sheimy," Localization of Wireless Sensor Networking using Bees Optimization Algorithm", IEEE International Symposium on ISSPIT, 2010, $15^{\text {th }}-18^{\text {th }}$ Dec., pp. 478-481.

[5] Akira Mutazono, Masashi Sugano, Masayuki Murata," Frog Call-Inspired Self-Organizing Anti-Phase Synchronization for Wireless Sensor Network",ISATTranscations on Computers and IntelligentSystems, vol 1, no. 2, pp. 86-93, Dec. 2009.

[6] Hui SUN, Jia ZHAO, "Application of Particle Sharing Based Particle Swarm Frog Leaping Hybrid Optimization Algorithm in Wireless Sensor Network Coverage Optimization”, Journal of Information \& Computational Science 8: 14, 2011, pp. 3181-3188. 
[7] Akira Mutazono, Masashi Sugano, Masayuki Murata, "EnergyEfficient Sleep Scheduling in Wireless Sensor NetworksInspired by Sattelite Behavior of Frogs", 8th IEEE International Conference on, vol., no., pp.450,455, March 292010 -April 22010

[8] Chandramouli H., Dr. Somashekar C Desai, K S Jagadeesh, Kashyap D Dhruve," Elephant swarm Optimization for Wireless Sensor Networks - A cross Layer Mechanism”, IJCET, Vol 4, Issue 2, 2013, pp. 45-60.

[9] RajaniMuraleedharan and Lisa Ann Osadciw, "Sensor Communication Network Using Swarm Intelligence", Proceedings of $2^{\text {nd }}$ IEEE upstate workshop on Sensor Networks, 2003, NewYork.

[10] Soumya Banerjee, CrinaGrosan, AjithAbrahamand P. K. Mahanti, "Intrusion Detection on Sensor Network Using Emotional Ant", $5^{\text {th }}$ International Conference on ISDA -2005.

[11] Yan Lianga1, Member, IEEE, JiannongCaob, Senior Member, IEEE, Lei Zhang b, Member, IEEE, RuiWangc, and QuanPana, Member, IEEE," A Biologically-Inspired Sensor Wakeup Control Method forWireless Sensor Networks",IEEE Transactions on Sysyem, Man and Cybernetics, Part C, vol 40, issue 5, pp. 525-538.

[12] S. S. Iyengar, Fellow, IEEE, Hsiao-Chun Wu.Senior member IEEE,N Balakrishnan, and Shih Chang, Member, IEEE, "Biologically Inspired Cooperative Routing for Wireless Mobiel Sensor Network", System journal, IEEE, vol 1, issue:1, 2007, pp. 29-37.

[13] Wang Chao, Senior member IEEE, LIN Quan,",Swarm Intelligence based Routing Algorithm for Wireless Sensor Network", IEEE International conference Neural Networks \& Signal Processing, Zhenjiang, China, June 8-10, 2008.

[14] SelcukOkdem, DervisKaraboga, "Routing in Wireless Sensor Networks using Ant Colony Optimization (ACO) Router Chip, Sensors ,2009, 9,909-921.

[15] Raghavendra V. Kulkarni, Senior Member, IEEE, Ganesh Kumar, Venayagamoorthy, Senior Member, IEEE,"Particle Swarm Optimization in Wireless Sensor Networks: A Brief Survey", IEEE Transactions on Systems, Man and Cybernetics-Part C : Applications and Reviews, vol. 41, No. 2, March 2011.

[16] Wen Hu, Van Nghia Tran, NirupamaBulusu, Chun Tung Chou, Sanjay Jha, and Andrew Taylor, "The Design and Evaluation of a Hybrid Sensor Network For Cane-toad Monitoring", Fourth International Symposium on Information processing in sensor networks, 2005, pp. 503-508.

[17] A XieHui, Zhang Zhi-gang, and NIE Feng "Novel Routing Protocol in Wireless Sensor Networks based on Ant Colony Optimization",International journal of Intelligent Information Technology Application, 2010, 3(1):1-5.

[18] Hong-Chi Shih, Shu-Chuan Chu, John F. Roddick, Mao-Hsiung Hung, Jeng-Shyang Pan, "Power Reduction of Wireless Sensor Networks Using Ant Colony Optimization", IEEE International Conference on CASN, $26^{\text {th }}-28^{\text {th }}$ Sept. 2010, pp. $464-467$.

[19] Faith Celik, AhmetZengin, SinanTuncel, "A Survey on Swarm Intelligence based routing protocols in Wireless sensor Networks, International journal of the Physical sciences vol. 5(14), November, 2010, pp. 2118-2126

[20] Joon-Woo Lee,Byoung-Suk Choi and Ju-Jang Lee, "Energy-Efficient Coverage of Wireless Sensor Networks Using Ant Colony Optimization with Three Types of Pheromones", IEEE Transactions on Industrial Informatics, vol.7, no. 3, August, 2011, pp.419427.

[21] Z. Pooranian, A. Barati and A. Movaghar. "Queen-bee Algorithm for Energy Efficient Clusters in Wireless Sensor Networks", World Academy of Science, Engineering and Technology, 73 2011, pp. 1080-1083. 